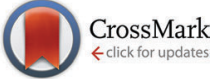

Cite this: Phys. Chem. Chem. Phys., $2014,16,25642$

Received 1st August 2014, Accepted 3rd October 2014

DOI: $10.1039 / c 4 c p 03436 h$

www.rsc.org/pccp

\title{
A theoretical study of the intramolecular charge transfer in 4-(dimethylamino)benzethyne $\dagger$
}

\author{
Javier Segarra-Martí $\neq^{* a}$ and Pedro B. Coto*b
}

\begin{abstract}
We have investigated the non-adiabatic relaxation processes occurring in the singlet manifold of 4-(dimethylamino)benzethyne (DMABE), a molecule isoelectronic with 4-(dimethylamino)benzonitrile (DMABN) but lacking its characteristic dual fluorescence, using multireference perturbation theory methods. The results obtained point out to the existence of a two-fold decay mechanism in which the population of the initially accessed $L_{a}$ state bifurcates towards a locally excited (LE) and a $\pi \sigma^{\star}$ state. Further relaxation to an emitting intramolecular charge transfer (ICT) state is impeded due to the presence of pronounced energy barriers along their associated potential energy surfaces. These results provide further evidence of the role of $\pi \sigma^{\star}$ states in the non-adiabatic relaxation processes of dialkylaminobenzonitriles.
\end{abstract}

\section{Introduction}

It has been over half a century since Lippert and co-workers ${ }^{1}$ reported for the first time the appearance of a second longwavelength band in the fluorescence spectra of 4-(dimethylamino)benzonitrile (DMABN, an electron donor-acceptor (EDA) molecule) in polar solvents. The authors assigned these bands to two different states. The so called normal band, that is also present in non-polar solvents, was assigned to the lowest-lying ${ }^{1} L_{b}$ state, also coined as the locally excited (LE) state. The new band appearing in polar solvents, sometimes called the anomalous band, was assigned to the polar and bright second excited state $\left({ }^{1} \mathrm{~L}_{\mathrm{a}}\right)$. This state would become the lowest-lying in polar solvents after reorganisation of the solvation shell, hence explaining the origin of the long-wavelength band. This model was soon challenged and since then, a long-standing effort has been carried out with the aim of understanding the molecular basis of this phenomenon. However, despite the large number of studies reported to date on this topic, the molecular mechanism underlying the dual fluorescence is still the focus of heated controversy, ${ }^{2-4}$ in part due to the complexity of the process that involves multiple electronic states and where solvent effects are all important, which makes its

\footnotetext{
${ }^{a}$ Instituto de Ciencia Molecular, Universitat de Valéncia, P.O. Box 22085, ES-46071 Valencia, Spain. E-mail: javier.segarra@uv.es; Fax: +34 963543274; Tel: +34963544428

${ }^{b}$ Institut für Theoretische Physik, Theoretische Festkörperphysik, Friedrich-Alexander-Universität Erlangen-Nürnberg, Erlangen D-91058, Germany. E-mail:pedro.brana-coto@physik.uni-erlangen.de

$\dagger$ Electronic supplementary information (ESI) available: Cartesian coordinates of all structures and plots of the active space orbitals. See DOI: $10.1039 / \mathrm{c} 4 \mathrm{cp} 03436 \mathrm{~h}$ \$ Present address: Dipartimento di Chimica “G. Ciamician”, Via Selmi 2, IT-40126 Bologna, Italy.
}

characterisation especially challenging from both theoretical ${ }^{5-8}$ and experimental ${ }^{9-12}$ standpoints.

Over the years, the vast majority of the studies on this topic have focussed on two main aspects, namely the nature and molecular structure of the state responsible for the anomalous fluorescence band and the mechanisms that lead to the population of this state after photoexcitation of DMABN.

The first of these issues seems to be largely settled and there is a wide consensus on the nature of the emissive intramolecular charge transfer (ICT) state, assumed to be responsible for the anomalous fluorescence feature. A majority of both theoretical ${ }^{13-15}$ and experimental ${ }^{10,11,16}$ studies follow the ideas of Grabowski and co-workers, ${ }^{6,17}$ who in their seminal work on the origin of the dual fluorescence on DMABN and related molecules proposed a model based on a twisted ICT state (TICT). In this model, the stabilisation of the ICT state is not only produced by the reorganisation of the polar solvent as in the model of Lippert et al., ${ }^{1}$ but also by the geometrical rearrangement of the molecule with the dimethylamino group characterised by a twisting of about $90^{\circ}$ with respect to the plane defined by the benzonitrile moiety. It is worthwhile mentioning, however, that this model has been challenged over the years and that different proposals exist. Among them, perhaps the most relevant is the planar ICT model (PICT). ${ }^{9,18-20}$ In this model, the second band appearing in the fluorescence spectra of DMABN and related molecules in polar solvents is assigned to a planar ICT state with no twisting of the dimethylamino moiety. However, the existence of a geometrical distortion of the dimethylamino group contributing to the reaction coordinate prompting the $\mathrm{La}\left(\pi \pi^{*}\right) \rightarrow$ ICT formation seems to be nowadays firmly established.

The studies centred on the photoinduced pathways connecting the initially excited $L_{a}\left(\pi \pi^{*}\right)$ state to the ICT species, on the other 
hand, have mainly focused on ICT formation from the LE state, as the rise time of the ICT fluorescence was found to be identical to the initial decay of the LE fluorescence. ${ }^{9}$ Recently, however, it has been suggested that a low-lying $\pi \sigma^{*}$ state arising from the promotion of an electron from the aromatic $\pi$ to a $\sigma^{*}$-like orbital localised on the cyano $(\mathrm{C} \equiv \mathrm{N})$ group in DMABN could be a major precursor of the ICT state. ${ }^{21-27}$ This idea is based on and influenced by previous $a b$ initio computational studies where a minimum in the $\pi \sigma^{*}$ ICT state was located. ${ }^{5,28,29}$ The involvement of $\pi \sigma^{*}$ states in different photoinduced processes has been increasingly recognised over the last few years and it is nowadays accepted that this kind of excited state plays an important role in processes ranging from molecular photodissociation to ICT formation. ${ }^{25,30-32}$ Two different types of processes arising from $\pi \sigma^{*}$ states have been widely reported over the years, one focused on $\mathrm{X}-\mathrm{H}$ dissociations in which $\sigma^{*}$ is of the $3 \mathrm{~s}$ Rydberg character. This corresponds to processes that undergo photodissociation leading to the loss of hydrogen. ${ }^{30}$ The other depicts processes in which the $\sigma^{*}$ orbital is placed in a triple bond and thus promotes the rehybridisation from sp to $\mathrm{sp}^{2}$, as is the case in the present study. For the case of DMABN and some related molecules, the evidence of the participation of $\pi \sigma^{*}$ states in the relaxation processes leading to the population of ICT states rests upon recent experimental studies carried out by Lim, Gustavsson and co-workers. ${ }^{24}$ The results obtained by these authors using time resolved transient absorption and fluorescence up-conversion experiments point out to the existence of two different relaxation pathways for the initial excited population of DMABN. Specifically, the decay time of the LE state was found to be similar to the rise time of the TICT transient absorption, and different from the ICT fluorescence feature measured by fluorescence up-conversion techniques, pointing towards the existence of more than one ICT state present in the molecule on polar solvents. In this context, the assigned rise time for the TICT-state transient registered at $425 \mathrm{~nm}$ is found to be the same as the decay time of the $700 \mathrm{~nm}$ transient measured of $\pi \sigma^{*}$ nature,$^{23,27}$ hinting towards the mediating role of $\pi \sigma^{*}$ in the process. This experimental evidence therefore suggests that the fluorescent ICT state is of a different nature than the TICT state probed by transient absorption, a suggestion that has been corroborated by means of $a b$ initio multireference perturbation theory computations, ${ }^{27}$ where it is shown that the low-lying $\pi \sigma^{*}$ state finds a pathway towards ICT formation in polar solvents. The transient absorption signals are then assigned to the TICT state, whereas the ICT fluorescence is referred to a partially twisted $\left(\sim 54.4^{\circ}\right)$ ICT (pTICT) state, providing a more complicated rationalisation of the dual fluorescence process. ${ }^{6}$ It is worth noting that the $700 \mathrm{~nm}$ signal has also been suggested to emerge due to transients arising from the LE state. ${ }^{33-35}$

To gain more insight into the mediating role of $\pi \sigma^{*}$ states in the photoprocesses involving dialkylaminobenzonitriles, in the present study we have investigated the photoinduced relaxation processes of 4-(dimethylamino)benzethyne (DMABE) using $a b$ initio multireference perturbation theory methods. DMABE is isoelectronic with DMABN, but unlike the latter, it does not exhibit the characteristic dual fluorescence feature even in polar solvents, ${ }^{23}$ pointing directly towards either an absence of ICT formation in this species or just the lack of emission even if the state was to be populated. This feature is here rationalised through a two-fold mechanism, previously proposed for $\mathrm{DMABN},{ }^{27}$ in which a bifurcation of the initially populated bright $\mathrm{L}_{\mathrm{a}}\left(\pi \pi^{*}\right)$ state leads to a LE minimum yielding the normal fluorescence feature widely reported in the literature, ${ }^{23}$ whereas the paths towards the TICT/pTICT minima and subsequent fluorescence are hampered, preventing the characteristic ICT fluorescence band, due to the presence of sizeable energy barriers along the potential energy surfaces describing the reaction paths in this particular system. The results emphasise the relevance of $\pi \sigma^{*}$ states in order to rationalise the photoinduced relaxation processes of dialkylaminobenzonitriles.

\section{Computational details}

Complete Active Space Self-Consistent Field (CASSCF) and Complete Active Space second-order Perturbation Theory (CASPT2) methods have been used in this study as implemented in the MOLCAS-7 program package. ${ }^{36,37}$ Multiconfigurational wave functions have been built using all $\pi$-like bonding and antibonding orbitals of the benzethyne moiety, the $\sigma$-like bonding and antibonding orbitals of the $\mathrm{C}-\mathrm{C}$ bond joining the ethyne group to the benzene ring and the lone pair of the nitrogen atom that is part of the dimethylamino group. Therefore, the active space used comprised 14 electrons in 13 orbitals. Geometry optimisation has been carried out at the state average CASSCF level of theory using four roots and equal weights. All reported structures have been optimised in vacuo. Seven-roots equal weights state average CASSCF calculations were used to compute the vertical excitations at the CASPT2 level. A ten-roots equal weights state average CASSCF was employed to compute the Linear interpolations in internal coordinates. On top of the CASSCF reference wave functions, CASPT2 second-order perturbative corrections have been computed maintaining all core electrons in a frozen state during the perturbation step and using the zeroth-order Hamiltonian as implemented with no IPEA shift in MOLCAS-7. ${ }^{38-41}$ An imaginary level shift of $0.2 \mathrm{au}$ has been used to avoid the effects of intruder states. $^{42}$ An ANO-S basis set with contraction C,N[4s3p1d]/ $\mathrm{H}[2 \mathrm{~s} 1 \mathrm{p}]$ has been employed throughout. ${ }^{43}$ Solvent effects have been evaluated within the polarisation continuum model $(\mathrm{PCM})^{44-46}$ by performing single point computations on the in vacuo optimised structures. Oscillator strengths have been computed using the in vacuo CASSCF functions for the calculation of the transition dipole moments and the CASPT2 values for the vertical energies.

\section{Results and discussion}

Following previous work, ${ }^{27}$ we have characterised the states relevant for the photoinduced ICT formation process in DMABE. These are the ground state (GS), the LE state and three different 

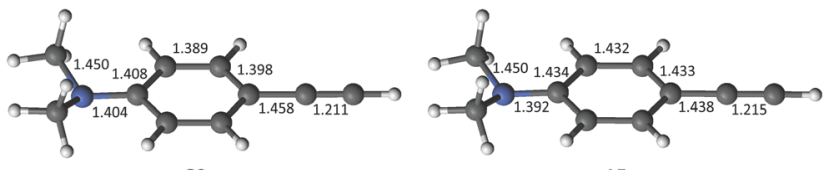

GS

LE
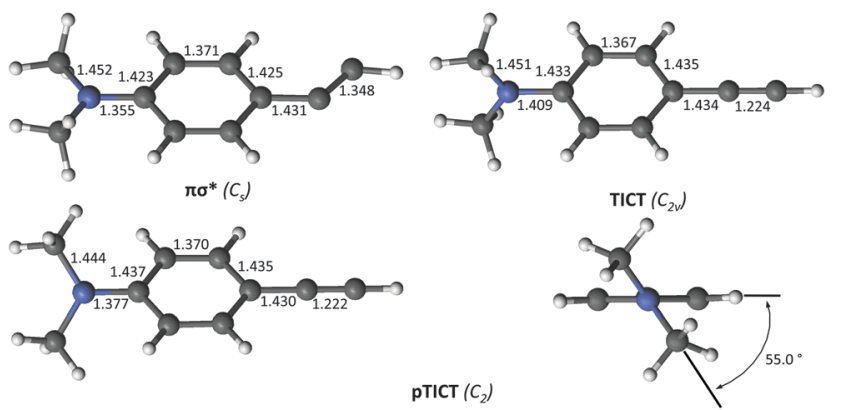

Fig. 1 Equilibrium structures of the different electronic states involved in the photophysics of DMABE investigated in this work. GS is the $S_{0}$ equilibrium structure. LE is the $S_{1}$ locally excited state. $\pi \sigma^{*}$, TICT and PTICT are different $S_{1}$ intramolecular charge transfer states. Their main geometric parameters are also shown. The point group symmetry used in the optimisation of the structures is given in parentheses when appropriate.

ICT states $\pi \sigma^{*}$, TICT, and pTICT, depicted in Fig. 1 together with their main geometrical parameters.

All structures exhibit a planar aromatic ring. However, they show substantial differences in the $\mathrm{C}-\mathrm{C}$ bond distances in the ring, with some structures displaying a quinoid-like pattern. In particular, the LE minimum presents an elongation in the $\mathrm{C}-\mathrm{C}$ distances along the aromatic ring, going from $\sim 1.40$ to $\sim 1.43 \AA$, analogous to the LE minimum previously reported for $\mathrm{DMABN},{ }^{27}$ and reminiscent of the locally excited planar minimum widely featured for DNA/RNA pyrimidines. ${ }^{47,48}$ Depending on the twist angle of the dimethylamino moiety we have identified two different twisted ICT states where the dimethylamino group acts as the donor and the benzethyne group as the acceptor, pTICT $\left(55^{\circ}\right)$ and TICT $\left(90^{\circ}\right)$. These structures show a slight elongation of the $\mathrm{C}-\mathrm{C}$ bonds adjacent to the twisting dimethylamino moiety. Finally, the last ICT state considered, the $\pi \sigma^{*}$ state, exhibits an optimised structure with a bent ethyne group with a $\mathrm{C} \equiv \mathrm{C}$ bond distance of $\sim 1.35 \AA$, which is close to usual $\mathrm{C}=\mathrm{C}$ distances. This structure is analogous to the rehybridised intramolecular charge transfer (RICT) state initially reported by Sobolewski and Domcke ${ }^{5,28,29}$ and subsequently featured in other computational studies ${ }^{49}$ as well as the $\pi \sigma^{*}$ structure previously described in ref. 27 for DMABN, making use of the CASPT2 method in all of the above.

Vertical excitation energies from the GS to all the relevant states involved in the photophysics of DMABE are displayed in Table 1. As can be seen, the excitation energies of the $L_{b}\left(\pi \pi^{*}\right)$ and $\mathrm{L}_{\mathrm{a}}\left(\pi \pi^{*}\right)$ states are close-lying, in agreement with our previous work $^{27}$ and other theoretical studies on DMABN, ${ }^{50,51}$ becoming practically degenerate in polar solvents. Solvation affects the $\mathrm{L}_{\mathrm{a}}\left(\pi \pi^{*}\right)$ state, while the $\mathrm{L}_{\mathrm{b}}\left(\pi \pi^{*}\right)$ state is virtually insensitive to it (see Table 1). This is a consequence of the different nature of these states and it can be readily noticed in the values of their respective dipole moments. This solvation
Table 1 Vertical excitation energies (eV) from the ground state, dipole moments (Debye) in vacuo and in solution, and oscillator strengths of the low-lying excited states of DMABE, computed at the CASPT2// CASSCF $(14,13) / A N O-S-V D Z P$ level of theory

\begin{tabular}{lcclcc}
\hline State & $\Delta E_{\text {vac }}$ & $|\mu|_{\text {vac }}{ }^{a}$ & $\Delta E_{\text {solv }}$ & $|\mu|_{\text {solv }}{ }^{a}$ & $f_{\text {solv }}$ \\
\hline $\mathrm{L}_{\mathrm{a}}\left({ }^{1} \pi \pi^{*}\right)$ & 4.46 & 7.97 & 4.27 & 11.41 & 0.474 \\
$\mathrm{~L}_{\mathrm{b}}\left({ }^{1} \pi \pi^{*}\right)$ & 4.18 & 2.07 & 4.18 & 2.98 & 0.004 \\
${ }^{a}$ Modulus of the dipole moment. & & & \\
\multicolumn{5}{l}{ M }
\end{tabular}

Table 2 Adiabatic (non-vertical) energies (eV) and dipole moments (Debye) of the low-lying excited states of DMABE in vacuo and adiabatic energies in solution (acetonitrile) computed at the CASPT2// CASSCF(14,13)/ANO-S-VDZP level of theory

\begin{tabular}{lcccr}
\hline State & $\Delta E_{\mathrm{vac}}{ }^{a}$ & $|\mu|_{\text {vac }}{ }^{b}$ & $\Delta E_{\text {solv }}{ }^{a}$ & $|\mu|_{\text {solv }}{ }^{b}$ \\
\hline LE & 3.94 & 2.15 & 3.92 & 2.65 \\
$\pi \sigma^{*}$ & 4.01 & 7.98 & 3.46 & 18.01 \\
pTICT & 3.96 & 9.77 & 3.64 & 13.52 \\
TICT & 4.03 & 11.73 & 3.45 & 15.93 \\
${ }^{a}$ Relative energy to the GS. ${ }^{b}$ Modulus of the dipole moment.
\end{tabular}

effect brings the absorbing ( $c f$. Table 1 ) $\mathrm{L}_{\mathrm{a}}$ close to the $\mathrm{L}_{\mathrm{b}}$ state, thus favouring the population of the latter non-adiabatically after photoexcitation. These results are also in agreement with previous computations where more complex solvent models built dynamically with explicit inclusion of solvent molecules were employed for DMABN. ${ }^{52}$

Table 2 contains the CASPT2//CASSCF adiabatic relative energies of all states involved in the photophysics of the DMABE considered in this work, both in vacuo and in solution. The effect of the solvent on the energies of the different states considered is especially prominent in the CT states, namely the $\pi \sigma^{*}$, pTICT, and TICT states, as is expected given their large dipole moments ( $c f$. Table 2). A large increase of the modulus of the dipole moment of these states takes place upon solvation, specially noticeable for the $\pi \sigma^{*}$ state, which goes from 7.98 to 18.01 Debye, but also relevant for both pTICT (9.77 to $13.52 \mathrm{D})$ and TICT (11.73 to $15.93 \mathrm{D})$ states.

As shown in Table 2, upon solvation all polar states lie close in energy (with differences smaller than $0.2 \mathrm{eV}$ ) and are significantly more stable than the LE state. This does not imply, however, that they are all accessible in the same manner upon excitation as it will depend on the energy barriers that the initially populated state, $\mathrm{L}_{\mathrm{a}}\left(\pi \pi^{*}\right)$, has to surmount after photoexcitation. In order to get an estimate of these energy barriers for the different possible deactivation channels of $\mathrm{L}_{\mathrm{a}}\left(\pi \pi^{*}\right)$, we have performed different linear interpolations in internal coordinates (LIICs, which provide an upper bound for the energetic barriers ${ }^{53}$ ) between the different states investigated in this work, depicted in Fig. 2. In particular, we have found that the $L_{a}$ and $L_{b}$ states are near degenerate at the FC region and towards the LE state. Furthermore, moderate energy barriers are found in the LIICs connecting the LE and the ICT states (pTICT and TICT, $\sim 0.3 \mathrm{eV})$. A similar energy barrier $(\sim 0.4 \mathrm{eV})$ has been obtained by connecting the initially accessed ICT 

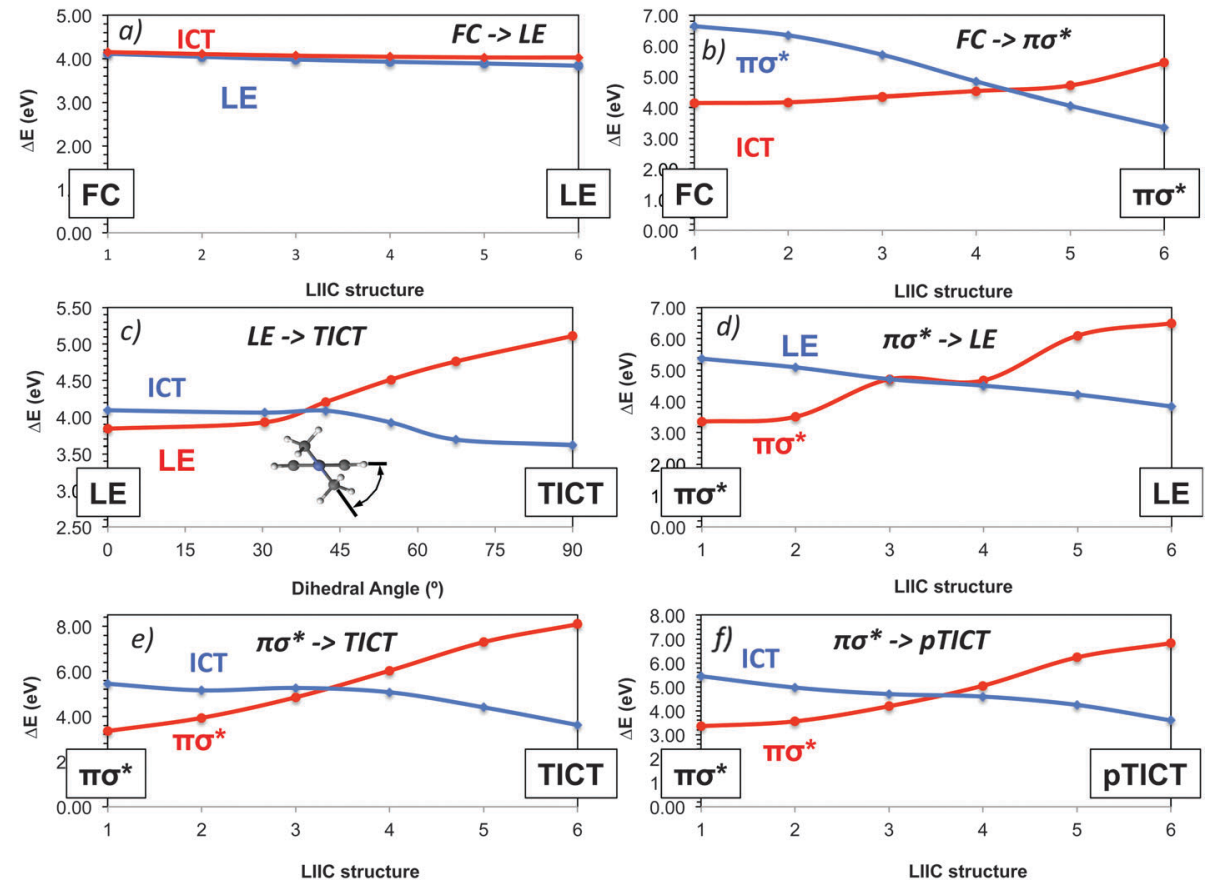

Fig. 2 Linear interpolation in internal coordinates (LIICs) between the different critical points characterised in the present study: (a) LIIC between the FC region and the LE minimum, (b) LIIC connecting the FC region and $\pi \sigma^{*}$ states, (c) LIIC between the LE and TICT states based on the distortions in the dihedral angle, (d) LIIC between the $\pi \sigma^{*}$ and LE states, (e) LIIC between the $\pi \sigma^{*}$ and TICT states and (f) LIIC connecting the $\pi \sigma^{*}$ and the pTICT states.

$\mathrm{L}_{\mathrm{a}}$-like state with the $\pi \sigma^{*}$ state between the FC region and the LE minimum. Fig. 2 therefore shows that the LIICs connecting the FC region with the TICT and $\pi \sigma^{*}$ states present a slight energy barrier that has to be surmounted in order to populate these states. For the TICT state, the profile presents similarities to previously reported sloped conical intersections, ${ }^{54}$ in which the population funnelling through the crossing is slightly hampered due to the steep nature of the state carrying the population towards the intersection. On the other hand, the flat profile shown by the LIIC connecting with the $\pi \sigma^{*}$ state is more similar to a peaked conical intersection, where the population is quickly transferred to the higher excited state, thus providing a more likely deactivation channel. Once populating the $\pi \sigma^{*}$ state, a barrierless decay towards its minimum is expected and supported theoretically, given the sizeable energy barriers of $\sim 1 \mathrm{eV}$ or higher present for the routes bridging the $\pi \sigma^{*}$ and $\mathrm{pTICT} / \mathrm{TICT} / \mathrm{LE}$ states that rationalise the trapped nature of the $\pi \sigma^{*}$ state and hence its long-lived lifetimes and subsequent probing through transient absorption. All this indicates that the population of TICT-like states is unlikely and agrees with the experimental evidence that shows no ICT fluorescence in the DMABE system as opposed to DMABN. ${ }^{23}$ Moreover, the TICT/pTICT minima present exceedingly small oscillator strengths associated with their respective transitions, both for emission and transient absorption, as opposed to what was observed previously in DMABN ${ }^{27}$ where the pTICT presented a non-negligible oscillator strength related to the emission back to the ground state and the TICT displayed two moderate $\left(\pi \pi^{*} \leftarrow \pi \pi^{*}\right)$ transient absorption bands as well as a strong $\left(\pi \sigma^{*} \leftarrow \pi \sigma^{*}\right)$ arising from a precursor $\pi \sigma^{*}$ state, rationalising the ICT fluorescence and transient absorption features arising from different states in agreement with the experiment. ${ }^{24,27}$ These results suggest a scenario for the photophysics of DMABE where the main protagonists are the LE and $\pi \sigma^{*}$ states.

More information on the role of the solvent in the stabilisation of the different electronic states involved in the process can be obtained using the Mulliken population analysis. Table 3 shows Mulliken charges localised in the phenyl, ethyne and dimethylamino moieties both in vacuo and in solution. As can be expected, the Mulliken charge distribution of both GS and LE states exhibits minor changes upon solvation. More significant changes are observed in the different CT states, in particular for the pTICT and $\pi \sigma^{*}$ states. Interestingly, the latter exhibits a different pattern of charge distribution compared to those of TICT and pTICT. For these, charges of different signs are localised in the neighbouring phenyl and dimethylamino groups (see Table 3) whereas for the $\pi \sigma^{*}$ state the positive and negative Mulliken charges are localised in the dimethylamino and ethyne moieties,

Table 3 In vacuo and in solution (acetonitrile) Mulliken charge distribution (in au) of the phenyl $(\mathrm{Ph})$, ethyne $(\mathrm{C} \equiv \mathrm{CH})$ and dimethylamino $\left(\mathrm{N}\left(\mathrm{CH}_{3}\right)_{2}\right)$ moieties for the different states investigated

\begin{tabular}{llllllll}
\hline & \multicolumn{3}{l}{ In vacuo } & & \multicolumn{3}{c}{ In solution } \\
\cline { 2 - 3 } System & $\mathrm{Ph}$ & $\mathrm{C} \equiv \mathrm{CH}$ & $\mathrm{N}\left(\mathrm{CH}_{3}\right)_{2}$ & & $\mathrm{Ph}$ & $\mathrm{C} \equiv \mathrm{CH}$ & $\mathrm{N}\left(\mathrm{CH}_{3}\right)_{2}$ \\
\hline $\mathrm{GS}$ & -0.1447 & -0.0353 & 0.1796 & -0.1530 & -0.0476 & 0.2006 \\
$\mathrm{LE}$ & -0.2533 & -0.0119 & 0.2652 & & -0.2772 & -0.0221 & 0.2993 \\
$\pi \sigma^{*}$ & -0.0825 & -0.3379 & 0.4204 & & -0.0034 & -0.6710 & 0.6676 \\
pTICT & -0.5322 & -0.1241 & 0.6564 & & -0.6117 & -0.2017 & 0.8133 \\
TICT & -0.7324 & -0.1268 & 0.8591 & & -0.7385 & -0.1393 & 0.8776
\end{tabular}


Table 4 Vertical excitation energies (eV) and oscillator strengths ( $f$ ) of the low-lying excited states of DMABE in vacuo and in solution calculated at the CASPT2//CASSCF $(14,13) / A N O-S-V D Z P$ level of theory

\begin{tabular}{lllll}
\hline Transition & $\Delta E_{\text {vac }}{ }^{a}$ & $f_{\text {vac }}$ & $\Delta E_{\text {solv }}{ }^{a}$ & $f_{\text {solv }}$ \\
\hline$\pi \pi^{*} \leftarrow \pi \pi^{*}$ & $2.07(599.0)$ & 0.001 & $2.11(587.6)$ & 0.001 \\
& $2.15(576.7)$ & 0.004 & $2.48(499.9)$ & 0.005 \\
& $1.90(652.6)$ & 0.012 & $1.53(810.4)$ & 0.010 \\
$\pi \sigma^{*} \leftarrow \pi \sigma^{*}$ & $1.86(667.0)$ & 0.041 & $1.89(656.0)$ & 0.041 \\
& $3.24(383.1)$ & 0.426 & $3.31(375.0)$ & 0.436 \\
& & &
\end{tabular}

respectively, indicating a more important role of the solvent in the stabilisation of this ICT state.

While the participation of the LE state in the photophysics of DMABE can be deduced from the emission spectra, the same cannot be argued for the dark $\pi \sigma^{*}$. To that end, we have computed the transient absorption spectra to investigate the population of the $\pi \sigma^{*}$ state during the DMABE non-adiabatic relaxation process in order to compare it with its reported experimental counterpart. ${ }^{23,26}$ For all systems investigated, we have found that only LE and $\pi \sigma^{*}$ show absorbing states with a sizeable oscillator strength within the range of energies investigated in the available experimental data. ${ }^{23,26}$ As reported in Table 4 , the LE state exhibits three $\left(\pi \pi^{*} \leftarrow \pi \pi^{*}\right)$ absorption bands with significant oscillator strengths associated with the following transitions, one with $f=0.005$ placed at $\sim 500 \mathrm{~nm}$, one with $f=0.001$ situated at $\sim 588 \mathrm{~nm}$ and another with $f=0.010$ placed at $\sim 810 \mathrm{~nm}$, which we tentatively assign to the broad band given at $\sim 530 \mathrm{~nm}$ and a slightly more intense shoulder placed at $\sim 600 \mathrm{~nm}$ in acetonitrile, respectively. ${ }^{23,26}$ The transient signal at $\sim 810 \mathrm{~nm}$ can be tentatively assigned as a contribution to the absorption band centred at $\sim 670 \mathrm{~nm}$ experimentally, previously reported in the literature for DMABN. ${ }^{33-35}$ In the case of the $\pi \sigma^{*}$ state, on the other hand, only one transition with significant oscillator strength $\left(\pi \sigma^{*} \leftarrow \pi \sigma^{*}, f=0.041\right)$ has been found within the experimental energy window reported in the literature, being placed at $\sim 656 \mathrm{~nm}$, which we assign to the experimental band with maximum absorption at $\sim 670 \mathrm{~nm}^{23,26}$ This signal appears in the same region as the previously discussed transient at $\sim 810 \mathrm{~nm}$ arising from the $\pi \pi^{*}$ state, yet the oscillator strength associated with the $\left(\pi \sigma^{*} \leftarrow \pi \sigma^{*}\right)$ transition is way larger so we assign this transition as the main contribution of the absorption maximum recorded at $\sim 670 \mathrm{~nm}$. It is worth mentioning that our calculations also predict the existence of a very intense $\left(\pi \sigma^{*} \leftarrow \pi \sigma^{*}\right)$ transient $(f=0.436)$ intrinsic to DMABE and not seen in DMABN at around $375 \mathrm{~nm}$. Further experimental efforts will be required to verify the existence of this absorption feature, which would represent a unique fingerprint for probing the presence of the $\pi \sigma^{*}$ state in DMABE and would further validate its population thus supporting its mediating role in the ICT formation.

Based on the results attained in this work, we propose a photophysical process like the one schematically represented in Fig. 3. Upon photoexcitation, the bright $\mathrm{L}_{\mathrm{a}}\left(\pi \pi^{*}\right)$ state is initially populated. This state subsequently relaxes via two different

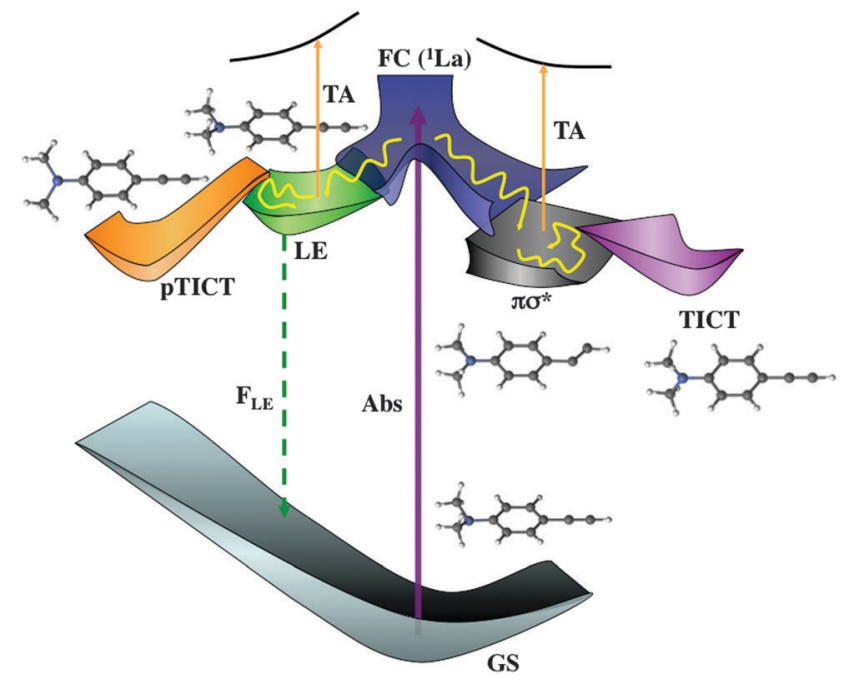

Fig. 3 Schematic representation of the photophysics of DMABE in acetonitrile.

pathways: on the one hand, part of the excited state population is expected to be funnelled towards the LE state, yielding the fluorescence feature observed experimentally. ${ }^{23}$ This state would also be responsible for the two $\left(\pi \pi^{*} \leftarrow \pi \pi^{*}\right)$ transient signals reported using femtosecond transient absorption spectra, ${ }^{23,26}$ which have been theoretically characterised and assigned in the present work. On the other hand, the bright $\mathrm{L}_{\mathrm{a}}\left(\pi \pi^{*}\right)$ state can also relax populating the $\pi \sigma^{*}$ state. This state would be responsible for the intense transient signal $\left(\pi \sigma^{*} \leftarrow \pi \sigma^{*}\right)$ observed at $\sim 656 \mathrm{~nm}$ ( $c f$. Table 4), in agreement with the experimental evidence, ${ }^{23,26}$ thus highlighting the role of the $\pi \sigma^{*}$ state in mediating the ICT formation process intrinsic to DMABE. The two twisted ICT states, pTICT and TICT, would not be populated during the relaxation process of DMABE due to the existence of moderate energy barriers along the reaction path. Put together, the results detailed above rationalise the absence of ICT fluorescence in DMABE while being consistent with the population of the $\pi \sigma^{*}$ state based on the interplay between the available transient absorption experimental data ${ }^{23,26}$ and the high-level $a b$ initio theoretical studies carried out in the present work.

\section{Conclusions}

We have carried out a theoretical investigation of the photophysics of DMABE using high-level ab initio multireference perturbation theory (CASPT2//CASSCF) methods. We have characterised the different states involved in the photoinduced ICT process of DMABE, namely the ground state, the LE state and three different ICT states (pTICT, TICT, and $\pi \sigma^{*}$ ). The results obtained are consistent with a two-fold decay mechanism where the initial photoinduced population of the absorbing $\mathrm{L}_{\mathrm{a}}\left(\pi \pi^{*}\right)$ state bifurcates leading to the population of the LE and $\pi \sigma^{*}$ states, in agreement with experimental data obtained using time-resolved transient absorption spectroscopy and previous 
theoretical models. Further non-adiabatic relaxation processes leading to the formation of the pTICT and TICT states are hampered due to the existence of moderate energy barriers along the reaction paths, therefore accounting for the lack of dual fluorescence in polar solvents exhibited by this molecule. The results obtained provide further evidence of the important role that $\pi \sigma^{*}$ states play in the rationalisation of the photophysics of DMABE and related dialkylaminobenzonitriles.

\section{Acknowledgements}

J.S.-M. acknowledges support from the Spanish MINECO project CTQ2010-14892. P.B.C. acknowledges financial support from projects CTQ2012-36966 (MINECO) and UAH2011/EXP-041 (UAH). We thank Dr T. Fujiwara and Prof. E. C. Lim for numerous interesting discussions.

\section{References}

1 E. Lippert, W. Lüder and H. Boos, in Advances in Molecular Spectroscopy, ed. A. Mangini, Pergamon, Oxford, 1962, p. 443.

2 J. Catalán, Phys. Chem. Chem. Phys., 2013, 15, 8811-8820.

3 K. A. Zachariasse, Phys. Chem. Chem. Phys., 2013, 15, 16976-16977.

4 J. Catalán, Phys. Chem. Chem. Phys., 2014, 16, 7734-7740.

5 A. L. Sobolewski and W. Domcke, Chem. Phys. Lett., 1996, 259, 119-127.

6 Z. R. Grabowski, K. Rotkiewicz and W. Rettig, Chem. Rev., 2003, 103, 3899-4031.

7 Y. Li, T. Liu, H. Liu, M.-Z. Tian and Y. Li, Acc. Chem. Res., 2014, 47, 1186-1198.

8 Y. Mercier and M. Reguero, Comput. Theor. Chem., 2014, 1040-1041, 212-218.

9 S. I. Druzhinin, N. P. Ernsting, S. A. Kovalenko, L. P. Lustres, T. A. Senyushkina and K. A. Zachariasse, J. Phys. Chem. A, 2006, 110, 2955-2969.

10 J. M. Rhinehart, R. D. Mehlenbacher and D. W. McCamant, J. Phys. Chem. B, 2010, 114, 14646-14656.

11 J. M. Rhinehart, J. R. Challa and D. W. McCamant, J. Phys. Chem. B, 2012, 116, 10522-10534.

12 M. Park, C. H. Kim and T. Joo, J. Phys. Chem. A, 2013, 117, 370-377.

13 C. Hättig, A. Hellweg and A. Köhn, J. Am. Chem. Soc., 2006, 128, 15672-15682.

14 L. Serrano-Andrés, M. Merchán, B. O. Roos and R. Lindh, J. Am. Chem. Soc., 1995, 117, 3189-3202.

15 I. Gómez, Y. Mercier and M. Reguero, J. Phys. Chem. A, 2006, 110, 11455-11461.

16 A. Pugliucci, E. Vauthey and W. Rettig, Chem. Phys. Lett., 2009, 469, 115-120.

17 Z. R. Grabowski, K. Rotkiewicz, A. Siemiarczuk, D. J. Cowley and W. Baumann, Nouv. J. Chim., 1979, 3, 443.

18 K. A. Zachariasse, S. I. Druzhinin, W. Bosch and R. Machinek, J. Am. Chem. Soc., 2004, 126, 1705-1715.
19 S. Cogan, S. Zilberg and Y. Haas, J. Am. Chem. Soc., 2006, 128, 3335-3345.

20 J. Dreyer and A. Kummrow, J. Am. Chem. Soc., 2000, 122, 2577-2585.

21 M. Z. Zgierski and E. C. Lim, J. Chem. Phys., 2004, 121, 2462-2465.

22 M. Z. Zgierski and E. C. Lim, J. Chem. Phys., 2005, 122, 111103.

23 J.-K. Lee, T. Fujiwara, W. G. Kofron, M. Z. Zgierski and E. C. Lim, J. Chem. Phys., 2008, 128, 164512.

24 T. Gustavsson, P. B. Coto, L. Serrano-Andrés, T. Fujiwara and E. C. Lim, J. Chem. Phys., 2009, 131, 031101.

25 M. Z. Zgierski, T. Fujiwara and E. C. Lim, Acc. Chem. Res., 2010, 43, 506-517.

26 T. Fujiwawa, M. Z. Zgierski and E. C. Lim, Phys. Chem. Chem. Phys., 2011, 13, 6779-6783.

27 P. B. Coto, L. Serrano-Andrés, T. Gustavsson, T. Fujiwara and E. C. Lim, Phys. Chem. Chem. Phys., 2011, 13, 15182-15188.

28 A. L. Sobolewski and W. Domcke, Chem. Phys. Lett., 1996, 250, 428-436.

29 A. L. Sobolewski and W. Domcke, J. Photochem. Photobiol., A, 1997, 105, 325-328.

30 A. L. Sobolewski, W. Domcke, C. Dedonder-Lardeuxc and C. Jouvet, Phys. Chem. Chem. Phys., 2002, 4, 1093-1100.

31 M. N. R. Ashfold, B. Cronin, A. L. Devine, R. N. Dixon and M. G. D. Nix, Science, 2006, 312, 1637-1640.

32 V. Ovejas, M. Fernández-Fernández, R. Montero, F. Castaño and A. Longarte, J. Phys. Chem. Lett., 2013, 4, 1928-1932.

33 K. A. Zachariasse, S. I. Druzhinin, S. A. Kovalenko and T. Senyushkina, J. Chem. Phys., 2009, 131, 224313.

34 I. F. Galván, M. E. Martín and M. A. Aguilar, Chem. Phys. Lett., 2010, 499, 100-102.

35 I. F. Galván, M. E. Martín and M. A. Aguilar, J. Chem. Theory Comput., 2010, 6, 2445-2454.

36 F. Aquilante, L. D. Vico, N. Ferré, G. Ghigo, P.-Å. Malmqvist, T. Pedersen, M. Pitonak, M. Reiher, B. O. Roos, L. SerranoAndrés, M. Urban, V. Veryazov and R. Lindh, J. Comput. Chem., 2010, 31, 224-247.

37 F. Aquilante, T. B. Pedersen, V. Veryazov and R. Lindh, Wiley Interdiscip. Rev.: Comput. Mol. Sci., 2013, 3, 143-149.

38 K. Andersson, P.-A. Malmqvist and B. O. Roos, J. Chem. Phys., 1992, 96, 1218-1226.

39 B. O. Roos, M. P. Fülscher, P.-Å. Malmqvist, M. Merchán and L. Serrano-Andrés, Adv. Chem. Phys., 1996, 93, 219-331.

40 G. Ghigo, B. O. Roos and P.-Å. Malmqvist, Chem. Phys. Lett., 2004, 396, 142-149.

41 D. Roca-Sanjuán, F. Aquilante and R. Lindh, Wiley Interdiscip. Rev.: Comput. Mol. Sci., 2012, 2, 585-603.

42 N. Forsberg and P.-Å. Malmqvist, Chem. Phys. Lett., 1997, 274, 196-204.

43 K. Pierloot, B. Dumez, P. O. Widmark and B. O. Roos, Theor. Chim. Acta, 1995, 90, 87-114.

44 J. Tomasi and M. Persico, Chem. Rev., 1994, 94, 2027-2094. 45 J. Tomasi, B. Mennucci and R. Cammi, Chem. Rev., 2005, 105, 2999-3094.

46 B. Mennucci, Wiley Interdiscip. Rev.: Comput. Mol. Sci., 2012, 2, 386-404. 
47 M. Merchán, R. González-Luque, T. Climent, L. SerranoAndrés, E. Rodríguez, M. Reguero and D. Peláez, J. Phys. Chem. B, 2006, 110, 26471-26476.

48 A. Giussani, J. Segarra-Martí, D. Roca-Sanjuán and M. Merchán, Top. Curr. Chem., 2013, DOI: 10.1007/128_2013_501.

49 I. Gómez, M. Reguero, M. Boggio-Pasqua and M. A. Robb, J. Am. Chem. Soc., 2005, 127, 7119-7129.

50 M. Chiba, T. Tsuneda and K. Hirao, J. Chem. Phys., 2007, 126, 034504.
51 P. Wiggins, J. A. G. Williams and D. J. Tozer, J. Chem. Phys., 2009, 131, 091101.

52 W. Sudholt, A. Staib, A. L. Sobolewski and W. Domcke, Phys. Chem. Chem. Phys., 2000, 2, 4341-4353.

53 L. Serrano-Andrés and J. J. Serrano-Pérez, Calculation of Excited States: Molecular Photophysics and Photochemistry on Display, in Handbook of Computational Chemistry, ed. J. Leszczynski, Springer-Verlag, 2012, ch. 14, pp. 483-560. 54 L. Blancafort, ChemPhysChem, 2014, 15, 3166-3181. 